\title{
SCALING OF OBSERVABLE PROPERTIES IN ROTATING STARS
}

\author{
D. Castañeda and R. G. Deupree \\ Institute for Computational Astrophysics and Department of Astronomy and Physics, Saint Mary's University, Halifax, \\ NS B3H 3C3, Canada; castaned@ap.smu.ca \\ Received 2014 April 11; accepted 2014 August 7; published 2014 September 18
}

\begin{abstract}
The spectral energy distribution as a function of inclination is computed using two-dimensional rotating stellar models and NLTE plane parallel stellar atmospheres. These models cover the range from $1.875 M_{\odot}$ to $3.0 M_{\odot}$. The deduced effective temperature is determined by $B-V$ computed from the spectral energy distribution, and the deduced luminosity is computed as the integral of the spectral energy distribution over all frequencies, assuming the distance and reddening are known. These deduced quantities are obtained from the observed spectral energy distribution assuming the objects are spherically symmetric, and thus the results are dependent on the inclination. Previous work has shown that the surface properties between two rotating stellar models with the same surface shape scale, and this is also true for the deduced effective temperature and luminosity over this limited mass range.
\end{abstract}

Key words: stars: atmospheres - stars: fundamental parameters - stars: rotation

\section{INTRODUCTION}

Advances in the general study of rotating stars have been limited by both theoretical and observational difficulties. A key example is that fundamental properties such as the effective temperature $\left(T_{\text {eff }}\right)$ and luminosity $(L)$ that one would deduce from observations now depend significantly on the angle of inclination $(i)$ between the line of sight and the star's rotation axis for sufficiently rapidly rotating stars (e.g., Collins \& Harrington 1966; Hardorp \& Strittmatter 1968; Maeder \& Peytremann 1970). This greatly complicates the determination of the star's position on the H-R diagram and hence nearly all other useful information unless its inclination can be determined.

Given a grid of plane parallel model atmospheres and a rotating model with latitudinal variation in the surface effective temperature and effective gravity, one can compute the spectral energy distributions (SEDs) for any inclination by performing a weighted integral of the intensity in the direction of the observer over the visible surface of the star (e.g., Slettebak et al. 1980; Linnell \& Hubeny 1994; Frémat et al. 2005; Lovekin et al. 2006). Integration of the flux in the SED over wavelength produces what we will refer as deduced $L$, and the application of nonrotating color-effective temperature relations to the SED produces what we will denote as deduced $T_{\text {eff }}$. Both these quantities will be strongly inclination dependent for sufficiently rapid rotation. There is also no straightforward relationship with the actual $L$ (i.e., the total amount of energy coming out of the star per unit time) or the actual $T_{\text {eff }}$, which we define as $(L /(A \sigma))^{1 / 4}$, where $A$ is the surface area of the star and $\sigma$ is the Stefan-Boltzmann constant. Naturally, the comparison between a computed SED and one observed has much prospect of success only if the inclination and the oblateness of the star are known. Fortunately, important advances in interferometric instrumentation over approximately the last decade have permitted resolved observations of some nearby rapid rotators (e.g., van Belle et al. 2001; Domiciano de Souza et al. 2003; Aufdenberg et al. 2006; Monnier et al. 2007; Zhao et al. 2009; Che et al. 2011). The direct process requires performing the calculations with different models until the observed SED properties are matched to the extent possible. This can be laborious and it would be far preferable to be able to start with the observed SED properties and work backward to what the luminosity and the latitudinal variation of the effective temperature must be. A crucial first step in this process is to demonstrate that there is a well-defined relation between the deduced quantities and their physically significant counterparts, even though this relation depends on both the inclination and the amount of rotation. We explore this relationship in this work.

Recently, Deupree (2011) showed that a number of properties of rotating models, particularly the surface effective temperature as a function of latitude, is proportional between models as long as the surface shape remains the same. Of course the surface radius as a function of latitude scales by definition. For the surface shapes to be exactly the same, the two models must have the same rotation law to within a multiplicative constant. The independence of latitude for the actual effective temperature and radius ratios suggests that observable properties such as the deduced luminosity and deduced effective temperature as functions of inclination may scale as well. If true, one might be able to at least place constraints on models and parameters that could produce the observed properties. It would also allow stepping backward from the observed properties to the actual luminosity and effective temperature in a straightforward way for cases in which both the inclination and surface shape are known. Being able to reduce the uncertainty in a star's actual properties is important in determining whether differences between observed and computed oscillation frequencies are due to not having a model with the correct actual properties or not having the interior model structure right. This is very timely because the discovery of multiple oscillation frequencies in rapidly rotating stars such as $\alpha$ Oph (Monnier et al. 2010), for which interferometric observations (Zhao et al. 2009) have been made, provides our best opportunity at the moment to study in detail the interior structure of rotating stars (e.g., Deupree et al. 2012; Mirouh et al. 2013).

We develop the model scaling relationships and discuss how the deduced properties scale in the next section. The following sections provide an example of how these scaling properties can be used and what are the limitations of the method.

\section{SCALING PROPERTIES}

We wish to examine the surface relationships between models that have the same surface shape. We assume that the surface 
is an equipotential, requiring the rotation law to be conservative. Some of the assumptions we make in the analysis hold for the laws we consider, and it is not clear what deviations from a conservative law are allowed before none of the scaling results hold. We shall return to this point in the final section.

\subsection{Scaling of Model Properties}

We first consider what scaling in this context means. Essentially, it means that for any surface variable of two models $\left(y_{1}\right.$, $y_{2}$ ) with the same shape,

$$
\frac{y_{2}\left(\theta_{j}\right)}{y_{1}\left(\theta_{j}\right)}=c_{y} \quad \forall \quad j,
$$

where $\theta_{j}$ is the co-latitude and $c_{y}$ is a constant. This is true for the surface radius by definition.

Having the surface shape of two different models the same also imposes a number of conditions on those models. First, models that have the same surface shape have the same rotation law form, except for an overall scale factor,

$$
\Omega_{2}\left(X_{R}, \theta_{j}\right)=c_{\Omega} \times \Omega_{1}\left(X_{R}, \theta_{j}\right) \quad \forall \quad j,
$$

where $\Omega$ is the rotation rate, $c_{\Omega}$ is a constant and $X_{R}=r_{R_{1}} / R_{1}=$ $r_{R_{2}} / R_{2}$.

The scaling of the rotation rate and the surface radius between two models with the same surface shape means the rotational velocities will scale as well. The scale factor can be determined from the ratio of the surface equatorial velocities of the two models. With both the rotational velocity and radius scaling, the centrifugal force scales as well.

Letting $\Phi$ denote the gravitational potential, we can write the total equipotential $(\Psi)$ at the surface in the following way:

$$
\begin{aligned}
\Psi_{1} & =\Phi_{1}(\theta)+\Omega_{1}^{2}(\theta) R_{1}^{2}(\theta) \frac{\sin ^{2}(\theta)}{2} \\
& =\frac{\Psi_{2}}{c_{\Psi}}=\frac{1}{c_{\Psi}}\left[\Phi_{2}(\theta)+\Omega_{2}^{2}(\theta) R_{2}^{2}(\theta) \frac{\sin ^{2}(\theta)}{2}\right] \\
& =\frac{1}{c_{\Psi}}\left[\Phi_{2}(\theta)+c_{\Omega}^{2} c_{R}^{2} \Omega_{1}^{2}(\theta) R_{1}^{2}(\theta) \frac{\sin ^{2}(\theta)}{2}\right] .
\end{aligned}
$$

Given that the gravitational potential will have only a small nonradial component, we see that this equation can be solved only if both terms are true individually. Thus,

$$
\Phi_{2}=c_{\Psi} \Phi_{1} \text { and } c_{\Psi}=c_{\Omega}^{2} c_{R}^{2}
$$

We see that the gravitational potentials scale as well. To the extent one can approximate the gravity at the surface by the Roche potential (which one can do quite well for all but the most rapid uniformly rotating models), one can obtain an estimate for the mass once we know the appropriate scaling constants.

Deupree (2011) has shown that scaling as defined in Equation (1) is true for the effective temperature for zero-age main sequence (ZAMS) models from 1.875 to $8 M_{\odot}$ with 20 different shapes. These effective temperature ratios appear to show a maximum variation of $0.5 \%$ over all latitudes. A part of the variation could be due to the fact that radii are discretized in the two-dimensional finite difference mesh - a zone is either inside the model or it is not. The surface at each latitude is taken to be the outer radial boundary of the zone, which the equipotential that describes the surface passes through.
We can see why the effective temperatures might scale by application of von Zeipel's (1924) law to two rotating homologous models that have the same shape. This means that the equipotential surfaces will have the same shape at the same fractional radius. Because the flux, assumed to be radiative, is perpendicular to the equipotential surface, one has

$$
\left[\frac{F_{\theta}\left(X_{R}, \theta_{j}\right)}{F_{r}\left(X_{R}, \theta_{j}\right)}\right]_{1}=\left[\frac{F_{\theta}\left(X_{R}, \theta_{j}\right)}{F_{r}\left(X_{R}, \theta_{j}\right)}\right]_{2},
$$

where the subscripts 1 and 2 refer to the two models. This means that the same fraction of the flux is being diverted from the radial direction for each of the two models. If this is true at all locations inside the model, then the distribution of the flux emerging from the model outer boundary must have the same relative distribution with latitude in both models. Because the effective temperatures are defined in terms of the flux emitted from a surface zone, the effective temperatures of the two models must scale, satisfying Equation (1).

We can also obtain this result from the work of Espinosa Lara $\&$ Rieutord (2011), which finds that one may write $T_{\text {eff }}=a g_{\text {eff }}^{\beta}$, where $\beta$ decreases slightly as the model becomes more oblate. Because $g_{\text {eff }}$ scales, then $T_{\text {eff }}$ scales as long as one is comparing models that have the same shape as we are here. We thank the referee for this insight.

Given sufficient information, one could expect to compute the latitude independent ratios of the surface radius, effective temperature, surface rotation velocity, and effective gravity. While these results may be of some theoretical interest, they would be more beneficial if properties obtained from observations, i.e., the deduced $T_{\text {eff }}$ and deduced $L$, also scaled.

\subsection{Scaling of Observed Properties}

Two key variables one wishes to obtain from a star are the actual $T_{\text {eff }}$ as defined above and $L$. This remains true for rotating stars, with the complication that neither the temperature nor the luminosity one would deduce from observations of a rapidly rotating star directly relate to intrinsic stellar properties because the deduced properties are strongly inclination dependent (e.g., Collins \& Harrington 1966; Hardorp \& Strittmatter 1968; Maeder \& Peytremann 1970; Gillich et al. 2008; Dall \& Sbordone 2011). To obtain a deduced effective temperature and luminosity from a SED for a rotating star requires the same knowledge about reddening and distance as for a spherical star, so we assume that this transformation can be performed to some degree and will address obtaining the deduced luminosity and effective temperature from dereddened SEDs with a known absolute flux.

The computation of the deduced effective temperature and luminosity as a function of inclination requires several steps. First, we must have the surface properties of the model, which here we take from the suite of ROTORC (Deupree 1990, 1995) ZAMS models computed by Deupree (2011). We note that these models force a relationship between the local effective temperature and the local surface temperature, unlike von Zeipel's law, which assumes that the surface is an equipotential (and hence constant temperature) surface while the effective temperature can vary significantly from pole to equator. The net effect is that the ROTORC models have a flatter relationship between the effective temperature and effective gravity, closer to 0.2 instead of the 0.25 of von Zeipel's law. We note that the behavior and values of our exponent with increasing rotation are quite 
similar to those of Espinosa Lara \& Rieutord (2011). Previous studies consistently find a lower value preferable (Monnier et al. 2007; Che et al. 2011; Claret 2012). Second, we must also have the intensities emerging from the surface for each member of a grid of stellar atmospheres. For the intermediate mass main sequence models we wish to explore, plane parallel model atmospheres are satisfactory. The model atmospheres are computed with the PHOENIX code (Hauschildt \& Baron 1999), and the grid covers the range in effective temperature from $7500 \mathrm{~K}$ to $11000 \mathrm{~K}$ in steps of $250 \mathrm{~K}$ and in $\log g$ from 3.333 to 4.333 in steps of 0.333 . The spectrum was computed from $600 \AA$ to $20000 \AA$, keeping a resolution greater than 250,000 below $8000 \AA$ and about 200,000 above $8000 \AA$. Lines in the four lowest ionization stages of $\mathrm{Al}, \mathrm{S}$, and $\mathrm{Fe}$; in the three lowest of $\mathrm{C}, \mathrm{N}$, $\mathrm{O}, \mathrm{Mg}, \mathrm{K}$, and $\mathrm{Ca}$; in the two lowest of $\mathrm{He}, \mathrm{Li}$, and $\mathrm{Na}$; and in the lowest of $\mathrm{H}$ and $\mathrm{Ne}$ are computed in NLTE. More specific details are given by Gillich et al. (2008) and Deupree et al. (2012). At lower temperatures than included we would need to include more species in NLTE, and at higher temperatures photometric temperature indicators in the visible region of the spectrum become harder to find. The net result of these two steps is that at any place on the surface of the rotating model, one can interpolate through the grid of model atmospheres in $\log T_{\text {eff }}$ and $\log$ $g$ to obtain the emergent intensity in any direction with respect to the local vertical. The third step calculates the direction to the observer, and thus the angle of the observer with respect to the local vertical, at every point on the surface, and performs the weighted integral over all the contributions of the intensities from every point on the surface visible to the observer to obtain the flux the observer would see. This approach for the third step is rather frequently used (e.g., Slettebak et al. 1980; Linnell \& Hubeny 1994; Frémat et al. 2005; Gillich et al. 2008; Aufdenberg et al. 2006; Yoon et al. 2008; Dall \& Sbordone 2011), and the specific details in our calculations are outlined by Lovekin et al. (2006). The final SEDs were obtained by using a $50 \AA$ wide boxcar filter. Because of this filtering and the fact that rotation does not affect the equivalent width, the Doppler shifts were not included in the flux integrals, making the flux calculation computationally "embarrassingly parallel." There is an option to include the Doppler shift when one wishes to compute specific line profiles with no filtering.

SEDs were obtained for uniformly rotating models for five masses $\left(1.875,2,2.25,2.5\right.$, and $\left.3 M_{\odot}\right)$ and six different rotation rates characterized by flatness $\left(1-R_{\mathrm{p}} / R_{\mathrm{eq}}\right)$ values of 0.112 , $0.134,0.156,0.180,0.207$, and 0.234 . To give an idea of how much rotation this is in more conventional terms, we note that the surface equatorial velocities range from about $230 \mathrm{~km} \mathrm{~s}^{-1}$ to about $360 \mathrm{~km} \mathrm{~s}^{-1}$. The most rapidly rotating model was chosen to keep the minimum effective temperature above $7500 \mathrm{~K}$, below which we would need to include other low ionization potential metals in NLTE. Models with slower rotation rates were not included because the pole to equator temperature variation was less than about $1000 \mathrm{~K}$. Some computed effective temperatures for the $1.875 M_{\odot}$ models for the two most oblate calculations were below this lower temperature limit, and those models were not included. The SEDs were computed at 10 equally spaced inclinations from pole on to equator on.

Both the deduced effective temperature and deduced luminosity were obtained from the computed SEDs. As expected, we found that $B-V$ provided a good indicator of the deduced effective temperatures, using a NLTE PHOENIX model of Vega with the parameters of Castelli \& Kurucz (1994) to calibrate the color indices. The deduced effective temperatures were obtained from

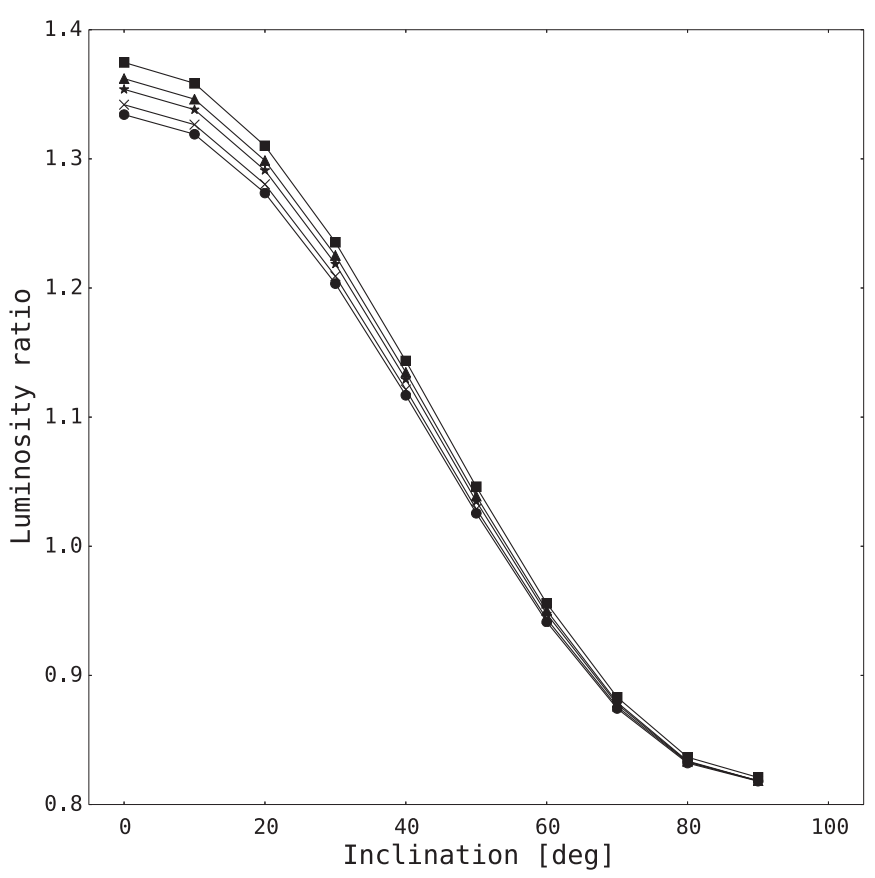

Figure 1. Ratio of the deduced luminosity to the luminosity as a function of inclination for ZAMS uniformly rotating models with masses of $1.875 M_{\odot}$ (circles), $2 M_{\odot}$ (crosses), $2.25 M_{\odot}$ (stars), $2.5 M_{\odot}$ (triangles), and $3 M_{\odot}$ (squares). The ratio of the polar radius to equatorial radius is 0.82 , corresponding to a surface equatorial velocity of about $300 \mathrm{~km} \mathrm{~s}^{-1}$.

the simulated $(B-V)$ color using the $(B-V)$-effective temperature relation for the plane parallel model atmospheres with the Vega calibration. The gravity used for the $(B-V)$-effective temperature relationship for the rotating models was the effective gravity at the co-latitude which corresponds to the inclination angle. However, the variation in effective gravity between the equator and pole is only a little larger than a factor of two, which would lead to a maximum error in the effective temperature of about $\pm 100 \mathrm{~K}$ based on a comparison of the change in the color-effective temperature relations with gravity for the plane parallel model atmospheres. The scaling should still be successful because the models at the same shape have the same effective gravity distribution.

The deduced luminosities were computed by integrating the computed flux over all wavelengths, including a Rayleigh-Jeans tail from the end of the calculated wavelengths to infinite wavelength, and multiplying the result by $4 \pi d^{2}$, where $d$ is an assumed distance to the model from the observer. Because the SED is inclination dependent, the deduced luminosity and effective temperature will be also. We also note that, because determining the gravity becomes part of the scaling algorithm if the inclination and shape are known, one can iterate the process to make the deduced gravity and the assumed gravity consistent.

We first turn to the deduced luminosities to determine how well they scale from one model with the same shape to another. For each model, we divide the deduced luminosity at each inclination by the actual luminosity. The results are presented in Figure 1 for models with the ratio of the polar to equatorial radius of 0.82 , the most rapidly rotating case for which the temperatures of all five masses fall within the range allowed. We see that the curves all have the same shape, but that the variation from pole to equator increases slightly as the mass increases, particularly at small inclination. While not perfect, the results in Figure 1 are sufficient to indicate that a reasonable 


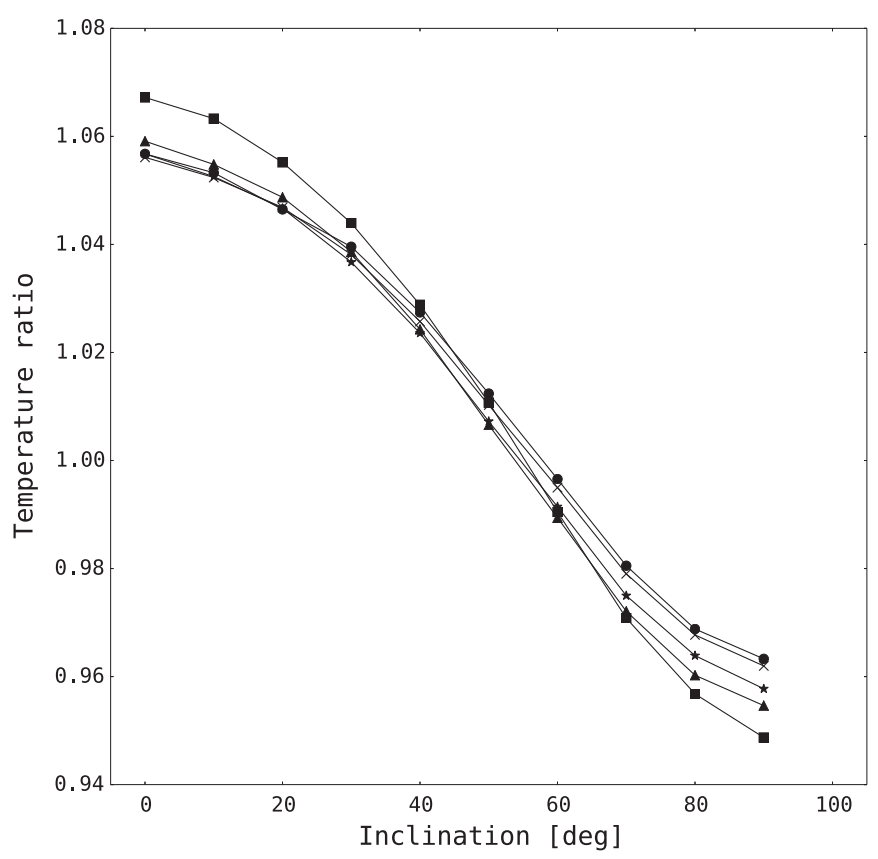

Figure 2. Ratio of the deduced effective temperature to the actual effective temperature (defined as the luminosity divided by the total surface area) as a function of inclination for the same models as in Figure 1. Note the general agreement, although the results for the $3 M_{\odot}$ suggest that there are limits to the applicability of the scaling.

determination of the intrinsic luminosity could be made given an observed luminosity, inclination, and polar to equatorial radius ratio (assuming uniform rotation), at least in the mass range covered.

For the deduced effective temperatures, we proceed in a manner similar to that used for the luminosity. Here the actual effective temperature, defined as the effective temperature obtained from the flux given by the actual luminosity divided by the total surface area of the model, plays the role that the actual luminosity played in the previous discussion. We take the ratio of the deduced effective temperature at each inclination divided by the actual effective temperature for each mass at a given shape. The results are shown in Figure 2. Again we see that the curves for different masses show the same form. Interestingly, the largest differences are shown for models seen equator on instead of pole on, except for the $3 M_{\odot}$ model, whose ratio at low inclination is noticeably larger than that for all the other masses. This variation with mass for both the deduced effective temperature and deduced luminosity suggests that these might be analogous to homology transforms for realistic models of stars-it works well over a restricted mass range, but is not universal and progressively degrades as the physical properties of the models become less similar. It is worth mentioning that the ratio of the model effective temperatures at a given latitude for these masses does not show any significant latitudinal variation, so that the variation in the deduced effective temperatures must originate in the conversion from physical effective temperatures to observed ones.

Finally, we consider whether the bolometric corrections deduced from these simulated SEDs are affected by any substantial changes rotation may introduce into the SED. This would be important if a full SED was not available. We computed the visual magnitude of our models and calculated $M_{V}$ using the assumed distance. The visual magnitude was calibrated by scaling the flux of our spherical model for Vega to match the observed

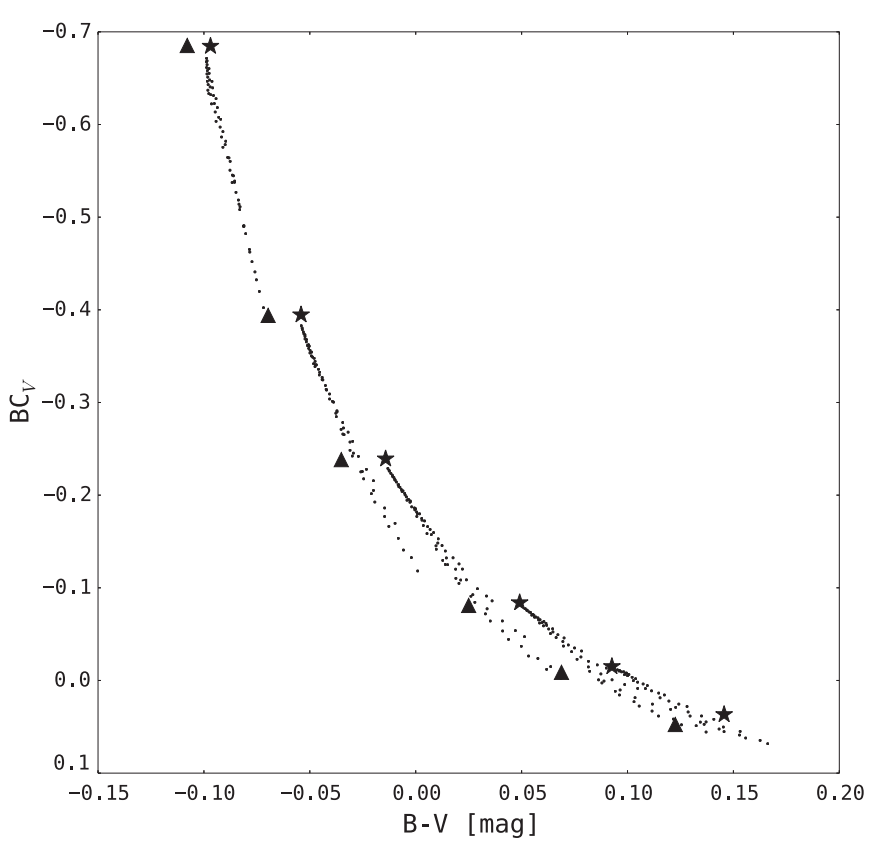

Figure 3. Dots show bolometric corrections for the SEDs of all models and inclinations. The stars denote plane parallel model atmospheres with $\log g=4.333$ and the triangles denote those with 4.000. Note that the bolometric corrections are not significantly different between the rotating models and the spherical models.

value above the earth's atmosphere at $5556 \AA$ (Hayes \& Latham $1975)$ and then integrating the flux in the $V$ filter and requiring $V=0.03$ mag (Bessell et al. 1998). The absolute bolometric magnitude comes directly from the model luminosity with the bolometric magnitude of the Sun set to 4.74. The bolometric corrections have been computed at inclinations between $0^{\circ}$ and $90^{\circ}$ in $10^{\circ}$ intervals for all models. The results are shown in Figure 3, a plot of the bolometric corrections as a function of $(B-V)$ for all inclinations of all models at all rotation speeds. Also shown in Figure 3 are the bolometric corrections for spherically symmetric models, the stars for $\log g=4.333$ and the triangles for $\log g=4.0$. Figure 3 indicates that the temperature is the key to the determination of the bolometric correction, but also that the effective gravity also plays a role (to about $0.07 \mathrm{mag}$ ). Except through the effective gravity, rotation by itself does not appear to produce any particular modifications to the bolometric corrections for these models.

\section{APPLICATION TO MODELS NOT ON THE ZAMS}

The scaling relationship for deduced luminosities and deduced $T_{\text {eff }}$ described above for the case of ZAMS models can be extended to models that are not in the same evolutionary state. To demonstrate this we consider a model of $\alpha$ Ophiuchus, a rapidly rotating A-type star. Interferometric observations of $\alpha$ Oph imply that it has a polar to equatorial radius ratio of 0.836 (Monnier et al. 2010) and $V \sin i$ in the range of $210-240 \mathrm{~km} \mathrm{~s}^{-1}$ (e.g., Bernacca \& Perinotto 1970; Abt \& Morrell 1995; Royer et al. 2002). We compared this model with two ZAMS models that have the same shape as $\alpha$ Oph: one has a similar mass but different actual $T_{\text {eff }}$ and the other with similar actual $T_{\text {eff }}$ to $\alpha$ Oph but different mass. A summary of the properties of each model is given in Table 1. Using as input the deduced luminosity and effective temperature at a specific inclination from the $\alpha$ Oph model and applying the scaling relations to each ZAMS model allows the determination of the deduced effective 


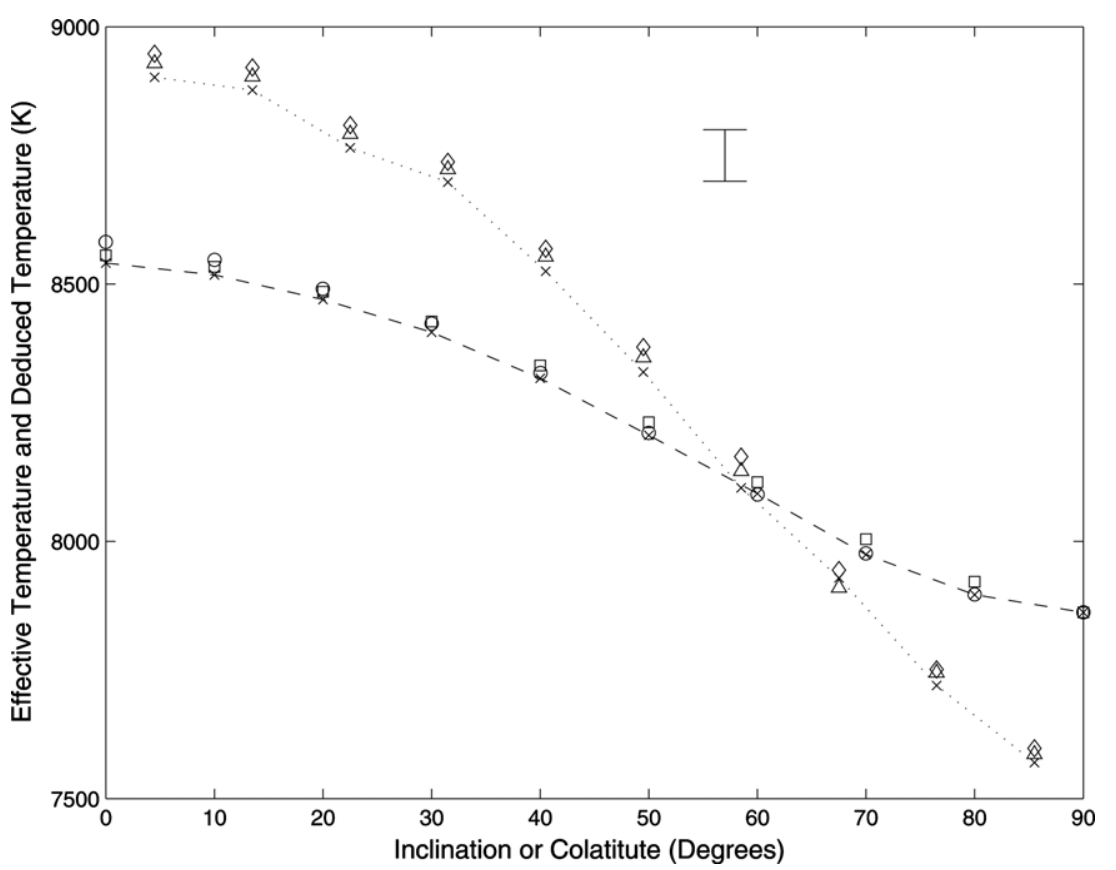

Figure 4. Plot of the effective temperature as a function of latitude (symbols on the dotted line) and the deduced effective temperature as a function of inclination (symbols on the dashed line). The diamonds, triangles, and crosses for the effective temperature refer to the results scaled from the $2.25 M_{\odot}$ ZAMS model, the results scaled from the $1.85 M_{\odot}$ ZAMS model, and the actual effective temperatures for the evolved model. The circles, squares, and crosses for the deduced effective temperatures refer to the results scaled from the $2.25 M_{\odot}$ ZAMS model, the results scaled from the $1.85 M_{\odot}$ ZAMS model, and the actual effective temperatures for the evolved model. A temperature range of $100 \mathrm{~K}$ is also indicated and it is clear that all three temperatures agree with each other for all cases considered.

Table 1

Model Properties

\begin{tabular}{lcccc}
\hline \hline Model & $\begin{array}{c}\text { Mass } \\
\left(M_{\odot}\right)\end{array}$ & $\begin{array}{c}V_{\text {eq }} \\
\left(\mathrm{km} \mathrm{s}^{-1}\right)\end{array}$ & $\begin{array}{c}\text { Actual } T_{\text {eff }} \\
(\mathrm{K})\end{array}$ & $\begin{array}{c}\text { Actual } L \\
(\mathrm{~K})\end{array}$ \\
\hline ZAMS 1 & 2.25 & 287 & 9474.5 & 25.48 \\
ZAMS 2 & 1.85 & 237 & 8187.9 & 11.71 \\
$\alpha$ Oph & 2.19 & 229 & 8122.8 & 32.63 \\
\hline
\end{tabular}

temperatures and luminosities at all inclinations, the effective temperature as a function of latitude, and finally the actual effective temperature and luminosity for the $\alpha$ Oph model. We can then compare the results predicted by the two ZAMS models with those for the $\alpha$ Oph model itself.

The comparisons of the deduced effective temperatures and actual effective temperatures are shown in Figure 4. We show the deduced temperatures as functions of inclination determined from the $\alpha$ Oph model (crosses), from the $2.25 M_{\odot}$ model (circles), and from the $1.85 M_{\odot}$ model (squares). Somewhat in keeping with the interferometric results for $\alpha$ Oph, we have chosen the deduced effective temperature and luminosity to be at an inclination of $90^{\circ}$. The latitudinal variation of the effective temperatures of the model is represented by the dotted line, and the variation of the deduced effective temperatures with inclination is shown by the dashed line. The same quantities are presented for the scaled $2.25 M_{\odot}$ and $1.85 M_{\odot}$ models. We note that in both cases each of the scaled two ZAMS models agree well with the actual model for $\alpha$ Oph. These results suggest that the precise details of the comparison model are not too important as long as the interior structures are sufficiently homologous. While "sufficiently homologous" is somewhat loosely defined, clearly these two models fit the requirements. On the other hand, one would not expect a $10 M_{\odot}$ main sequence star to be an appropriate model for either $\alpha$ Oph or for a $10 M_{\odot}$ red giant.
The result is the same for the deduced luminosity, as shown in Figure 5. Both ZAMS models scale well to the deduced luminosity for the $\alpha$ Oph model at all inclinations, although the $1.85 M_{\odot}$ ZAMS model agrees with the $\alpha$ Oph model a little better. As one might expect from the agreement of these features, the actual luminosity and the actual effective temperature for each ZAMS model agree to within $0.12 L_{\odot}$ and $40 \mathrm{~K}$ with the value of the $\alpha$ Oph model.

\section{SCALING ALGORITHM}

We have argued that the deduced luminosity and effective temperature scale for rotating models with the same shape over some limited range of conditions, and that this allows us to determine reasonable values for the luminosity and actual effective temperature of the unknown star from the models. Here we develop an algorithm to use the deduced and model scaling relations to obtain some intrinsic properties of a rotating star (for convenience, we shall refer to the rotating stellar models with known properties as the "model" and the unknown object whose properties we wish to obtain as the "star"). We start by assuming that we have a deduced effective temperature, deduced luminosity, and a measurement of $V \sin i$. For the moment we assume that we also have the shape and inclination for the star as well. The reference model must have the surface radius, effective temperature, surface rotational velocity, and surface effective gravity as functions of latitude, the deduced effective temperatures and luminosities as functions of inclination, and the mass and luminosity of the model.

The algorithm proceeds as follows: from $V \sin i$ and the inclination of the star, compute the surface equatorial velocity, $V_{\text {eq }}$. Compute the ratio of the surface equatorial velocity of the model and the star. Because Equation (1) is true for the surface velocity, one can compute the star's surface rotation velocity at all latitudes. Because both the deduced effective 


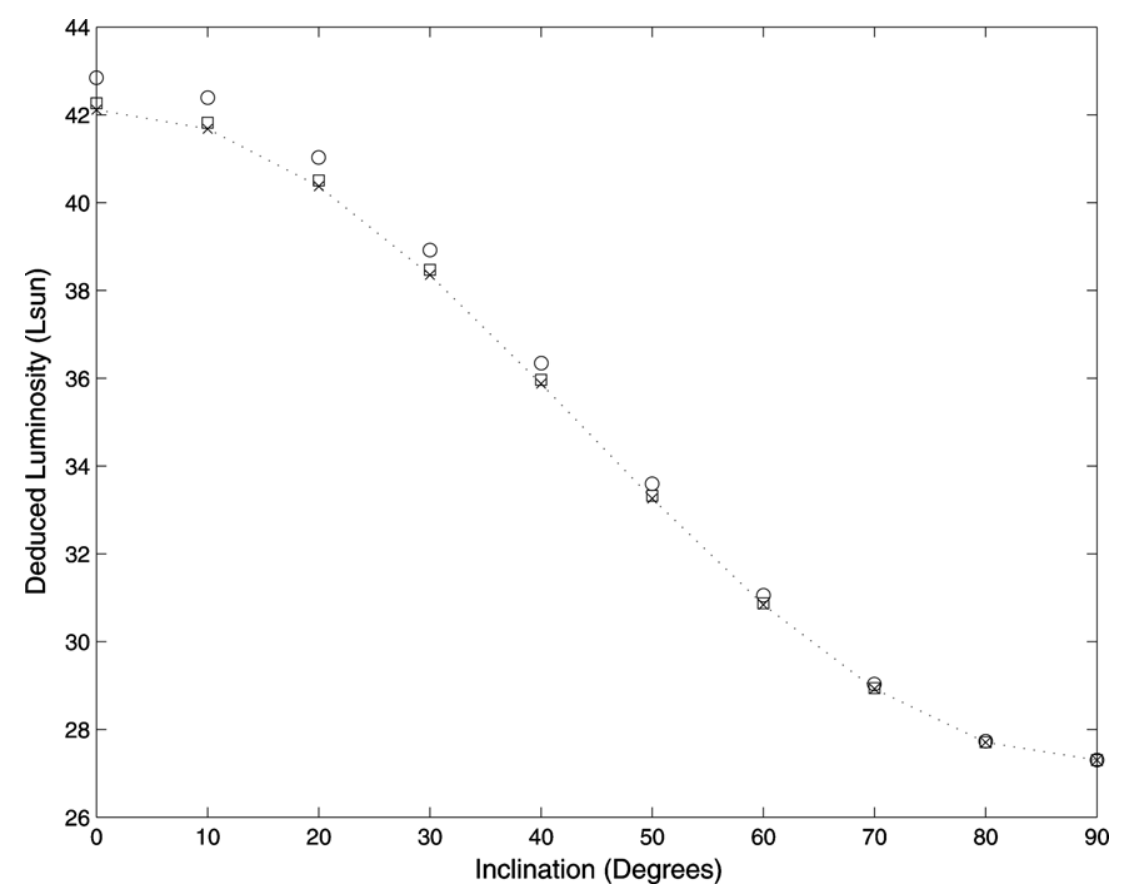

Figure 5. Deduced luminosity as a function of inclination for the evolved model based on the scaled $2.25 M_{\odot}$ ZAMS model (circles), the scaled $1.85 M_{\odot}$ ZAMS model (squares), and for the actual evolved model itself (crosses).

temperatures and the latitudinal effective temperatures scale as shown in Figure 4, we have

$$
\frac{T_{\mathrm{eff}, 1}\left(\theta_{j}\right)}{T_{\mathrm{eff}, 2}\left(\theta_{j}\right)}=\frac{T_{\mathrm{d}, 1}\left(i_{j}\right)}{T_{\mathrm{d}, 2}\left(i_{j}\right)}=\frac{T_{\mathrm{eff}, a, 1}}{T_{\mathrm{eff}, a, 2}} \quad \forall \quad j,
$$

where 1 refers to the model, 2 refers to the star, $\mathrm{d}$ refers to the deduced temperature, and $a$ refers to the actual $T_{\text {eff }}$ previously defined. Because the deduced temperatures are known at the inclination of the star for both the model (by interpolation) and the star (by observation), we can obtain the effective temperature at all latitudes and the actual effective temperature for the star. The actual luminosity of the star can be computed from the deduced luminosity at the assumed inclination, the deduced luminosity as a function of inclination for the model and the actual luminosity for the model. With the actual luminosity and actual effective temperatures known for both the star and the model, one can compute the ratio of the radii because the only difference between the surface areas of the model and the star is the difference in the radius. Hence, the radius of the star at every latitude of the star follows from the radius profile of the model.

The steps so far have depended only on the model, its deduced properties, and the scaling relations for both. These steps have resulted in the surface properties of the star as a function of latitude. The next step requires that the surface be an equipotential. As shown in Section 2.1, we use the fact that the gravitational part of the total potential and centrifugal potential must scale the same way for a given shape (i.e., at a given latitude, the ratio between the centrifugal potentials of the model and star and the ratio between the gravitational potentials of the model and the star must be the same). Because we have computed both the surface rotational velocity and the surface radius as functions of latitude for the star and have them for the model, we can obtain the ratio of the centrifugal potentials and hence of the gravitational potentials. Figure 6 presents a diagram of the information required from both the model and the star, as well as how the various unknowns of the star are determined from the scaling.

If we assume that the gravitational potential at the surface is given by that of a spherical star, at least at the equator, we can compute an estimate for the mass of the star. This assumption is generally good unless there is significant differential rotation where the material close to the rotation axis rotates much faster than the material farther away from the axis. It is certainly excellent for uniformly rotating models except those very near critical rotation (e.g., Ostriker \& Mark 1968; Faulkner et al. 1968; Jackson et al. 2004; Deupree 2011).

The reason for performing these last steps was to get an estimate of the mass, which could be used with the actual luminosity to provide a check on the results. All the models utilized here are core hydrogen burning objects for which the main sequence mass-luminosity law should hold. A check on the reasonableness of the assumed inclination and shape can be made through how well the derived mass and actual luminosity fit the mass-luminosity law.

\section{LIMITATIONS OF THE ALGORITHM}

The scaling we have described relies on certain assumptions, and it is reasonable to see to what extent they can be relaxed. We have assumed that the surface is an equipotential, which only exists if the rotation law is conservative. Even for conservative rotation laws, it remains an assumption that the surface is an equipotential. This likely matters for the part of the solution that makes an estimate of the mass, but it need not affect the scaling of the observable properties as long as whatever mechanism determines the surface shape determines it in the same way for both the unknown and comparison objects. Our very limited knowledge of the surfaces of rotating stars does not allow an answer to this question.

We also assume that we know both the inclination and the surface shape of the unknown object. This in general is not true, and it turns out that there are combinations of inclination and shape that produce reasonable results, including masses that fit 


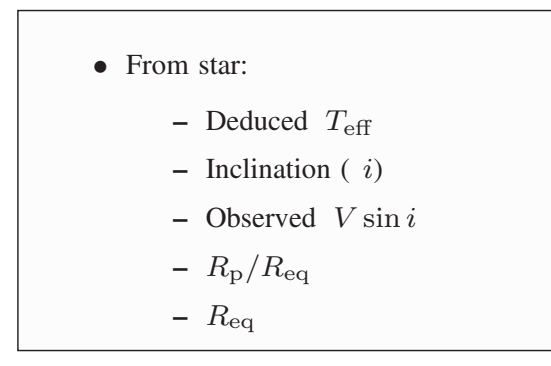

- From reference model:

- Same $R_{\mathrm{p}} / R_{\text {eq }}$ as star

- As function of colatitude:

* Surface radius

* Surface $T_{\text {eff }}$

* $\log g_{\text {eff }}$

* Rotational velocity $(V)$

- Known actual $L\left(L_{a c}\right)$ and actual $T_{\text {eff }}\left(T_{\text {eff,ac }}\right)$

- Deduced $T_{\text {eff }}$ for various inclinations

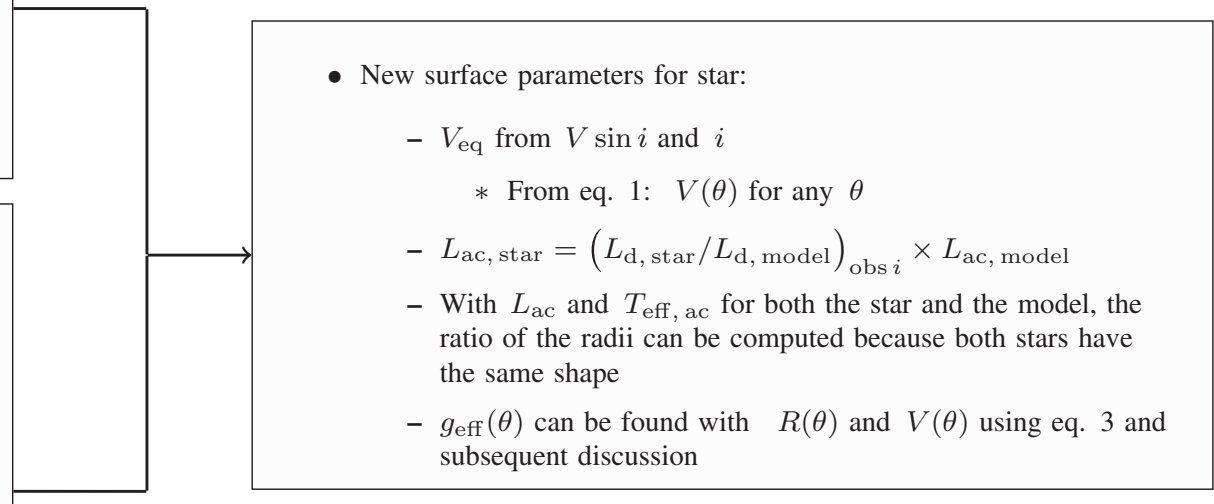

Observables can be calculated:

(+) SED

(+) Line profiles

(+) Synthetic interferometry

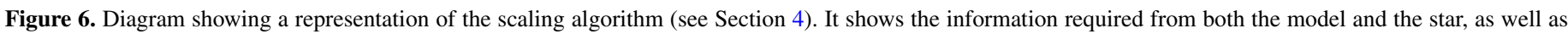
how the various unknowns of the star determined from the scaling are presented.

the mass-luminosity relation. The general trend is that more rapid rotation (i.e., more oblate shapes) can be offset by smaller inclinations. One might also add that determining the surface shape accurately potentially pays dividends by possibly placing constraints of the rotation law.

We have also used a single composition for all our models. A different composition would make a difference by producing a different color-effective temperature relation. We can obtain a crude estimate of how this might affect results by comparing the color-effective temperature relations of several spherical models at different temperatures with two different compositions. We calculated NLTE plane parallel model atmospheres with temperatures of 8000,9000 , and $10,000 \mathrm{~K}$ using half the metallicity of our previously computed models. The deduced temperatures for spherical models at these temperatures were all within $50 \mathrm{~K}$ of the actual temperature when using the color-effective temperature relation from the full metallicity models.

These results lead us to believe that scaling of observables can be a useful technique to make the bridge between what one observes for rotating stars and physically useful information under appropriate conditions. We should caution that these results cover only a limited range in gravity and effective temperature and that extension far outside this range may not be warranted.

The authors thank Compute Canada and ACEnet for the computational resources used in this research.

\section{REFERENCES}

Abt, H. A., \& Morrell, N. I. 1995, ApJS, 99, 135

Aufdenberg, J. P., Merand, A., du Foresto, V. C., et al. 2006, ApJ, 645, 664
Bernacca, P. L., \& Perinotto, M. 1970, CoAsi, 239, 1

Bessell, M. S., Castelli, F., \& Plez, B. 1998, A\&A, 333, 231

Castelli, F., \& Kurucz, R. L. 1994, A\&A, 281, 817

Che, X., Monnier, J. D., Zhao, M., et al. 2011, ApJ, 732, 68

Claret, A. 2012, A\&A, 541, A113

Collins, G. W., II, \& Harrington, J. P. 1966, ApJ, 146, 152

Dall, T. H., \& Sbordone, L. 2011, JPhCS, 328, 012016

Deupree, R. G. 1990, ApJ, 357, 175

Deupree, R. G. 1995, ApJ, 439, 357

Deupree, R. G. 2011, ApJ, 735, 69

Deupree, R. G., Castañeda, D., Peña, F., \& Short, C. I. 2012, ApJ, 753, 20

Domiciano de Souza, A., Kervella, P., Jankov, S., et al. 2003, A\&A, 407, L47

Espinosa Lara, F., \& Rieutord, M. 2011, A\&A, 533, A43

Faulkner, J., Roxburgh, I. W., \& Strittmatter, P. A. 1968, ApJ, 151, 203

Frémat, Y., Zorec, J., Hubert, A.-M., \& Floquet, M. 2005, A\&A, 440, 305

Gillich, A., Deupree, R. G., Lovekin, C. C., Short, C. I., \& Toqué, N. 2008, ApJ, 683,441

Hardorp, J., \& Strittmatter, P. A. 1968, ApJ, 151, 1057

Hauschildt, P. H., \& Baron, E. 1999, JCoAM, 109, 41

Hayes, D. S., \& Latham, D. W. 1975, ApJ, 197, 593

Jackson, S., MacGregor, K. B., \& Skumanich, A. 2004, ApJ, 606, 1196

Linnell, A. P., \& Hubeny, I. 1994, ApJ, 434, 738

Lovekin, C. C., Deupree, R. G., \& Short, C. I. 2006, ApJ, 643, 460

Maeder, A., \& Peytremann, E. 1970, A\&A, 7, 120

Mirouh, G. M., Reese, D. R., Lara, F. E., Ballot, J., \& Rieutord, M. 2013, in IAU Symp. 9, Precision Asteroseismology, ed. J. A. Guzik et al. (Cambridge: Cambridge Univ. Press), 455

Monnier, J. D., Townsend, R. H. D., Che, X., et al. 2010, ApJ, 725, 1192

Monnier, J. D., Zhao, M., Pedretti, E., et al. 2007, Sci, 317, 342

Ostriker, J. P., \& Mark, J. W.-K. 1968, ApJ, 151, 1075

Royer, F., Grenier, S., Baylac, M.-O., Gomez, A. E., \& Zorec, J. 2002, A\&A, 393, 897

Slettebak, A., Kuzma, T. J., \& Collins, G. W. 1980, ApJ, 242, 171

van Belle, G. T., Ciardi, D. R., Thompson, R. R., Akeson, R. L., \& Lada, E. A. 2001, ApJ, 559, 1155

von Zeipel, H. 1924, MNRAS, 84, 665

Yoon, J., Peterson, D. M., Zagarello, R. J., Armstrong, J. T., \& Pauls, T. 2008, ApJ, 681, 570

Zhao, M., Monnier, J. D., Pedretti, E., et al. 2009, ApJ, 701, 209 